\title{
Évaluation du risque de puits sec dans les réservoirs hétérogènes
}

\author{
L.Y. Hu' ${ }^{1}$ A. Haas $^{2}$ et L. Piacentino' \\ 1 Institut français du pétrole, 1 et 4, avenue de Bois-Préau, 92852 Rueil-Malmaison Cedex - France \\ 2 Elf Exploration \& Production, avenue Larribau, 64018 Pau - France \\ e-mail: l-ying.hu@ifp.fr - Andre.Haas@elf-p.fr - laurent.piacentino@ifp.fr
}

\begin{abstract}
Résumé - Dans le cas d'un réservoir hétérogène avec une forte proportion de faciès non (ou peu) perméables (faciès non-réservoir), le risque pour qu'un puits soit foré complètement dans les faciès nonréservoir peut être considérable. Le risque (ou la probabilité) du puits sec est défini comme une fonction (non linéaire) des paramètres du modèle stochastique de réservoir (proportions de faciès et leurs longueurs de corrélation) et des paramètres du puits (intervalle de production et orientation). Nous disposons d'une formule analytique de la probabilité du puits sec pour une classe de modèles stochastiques de réservoir ayant la propriété semi-markovienne (e.g. le modèle booléen à objets élémentaires convexes). Dans cet article, nous proposons une généralisation de cette formule à d'autres modèles stochastiques de réservoir couramment utilisés en pratique.
\end{abstract}

L'approche proposée est validée sur deux modèles de réservoir générés respectivement par les deux méthodes géostatistiques les plus utilisées, à savoir la méthode de simulation séquentielle d'indicatrices et la méthode de simulation gaussienne tronquée. La construction de ces modèles de réservoir est inspirée d'un champ réel. Dans les deux cas, l'approximation obtenue de la probabilité du puits sec est satisfaisante. Contrairement à un plan d'expériences conventionnel basé sur une approximation polynomiale, l'approche proposée confère une signification géologique aux paramètres qui influencent la probabilité du puits sec.

La méthode proposée peut être utilisée pour optimiser l'orientation et la complétion (intervalle de production) d'un nouveau puits. En particulier, elle peut être utilisée pour évaluer l'efficacité d'un puits horizontal par rapport à un puits vertical ou incliné. Lors d'une évaluation de la performance d'un nouveau puits, il convient d'évaluer d'abord le risque de puits sec et ensuite d'évaluer sa performance, sachant qu'il rencontre des faciès réservoir.

Mots-clés : hétérogénéité, incertitude, modèle booléen, simulation géostatistique, régression non linéaire, plan d'expériences.

\footnotetext{
Abstract - Evaluation of the "Dry Well" Risk in Heterogeneous Reservoirs - With heterogeneous reservoirs, there is a high risk of drilling wells in nonreservoir facies. An evaluation of this "dry well" risk is necessary for decision making. The "dry well" risk probability is defined as a nonlinear function of the stochastic reservoir model parameters (facies proportions and their correlation lengths) and the well parameters (production interval and orientation). There exists an analytical formula of the "dry well" risk probability for a class of stochastic reservoir models which have the semi-Markovian property (e.g. the Boolean model with convex elementary objects). This paper proposes a generalization of this formula to other stochastic reservoir models commonly used in practice.

The proposed approach is validated on two numerical reservoir models generated respectively by the most commonly used geostatistical methods: the sequential indicator simulation and the truncated
} 


\begin{abstract}
Gaussian simulation. The construction of these reservoir models is inspired from a real field. In both cases satisfactory approximations of the "dry well" risk probability are obtained. Unlike a conventional experimental design based on a polynomial approximation, the proposed approach gives a geological signification to the parameters which influence the "dry well" risk probability.

The method can be used to optimize the orientation and the completion (production interval) of a new well. In particular, it can be used to evaluate the efficiency of a horizontal well as opposed to a vertical or inclined well. When evaluating the uncertainty on the performance of a new well, it is convenient to evaluate first the "dry well" risk and then to evaluate the well performance provided that the well hits the reservoir facies.

Keywords: heterogeneity, uncertainty, Boolean model, geostatistical simulation, nonlinear regression, experimental design.
\end{abstract}

\section{INTRODUCTION}

L'approche stochastique est aujourd'hui couramment utilisée dans l'industrie pétrolière pour la caractérisation de réservoirs hétérogènes, car elle permet de prendre en compte les incertitudes dues à une connaissance partielle des réservoirs. Lorsqu'un modèle stochastique est choisi (selon des critères géologiques par exemple) pour représenter un réservoir, les incertitudes sur les réponses en production sont générées d'une part par l'incertitude sur les paramètres du modèle, d'autre part par la possibilité de créer une infinité de réalisations différentes de ce modèle. Par ailleurs, les paramètres d'exploitation (e.g. longueur et orientation d'un puits) influencent aussi les résultats de production. La quantification des incertitudes sur les réponses en production d'un puits doit tenir compte à la fois de l'incertitude sur les paramètres du modèle stochastique de réservoir, de la variation des paramètres d'exploitation et de la multiplicité des réalisations du modèle stochastique.

Dans le cas d'un réservoir hétérogène avec une forte proportion de faciès non (ou peu) perméables (faciès nonréservoir), la probabilité pour qu'un puits soit foré complètement dans les faciès non-réservoir peut être considérable. Par conséquent, la distribution d'une réponse en production ne peut être modélisée simplement par une loi gaussienne ou log-normale. En effet, dans un tel cas, la distribution d'une réponse en production est composée d'une probabilité à production nulle (dite probabilité du puits sec) et d'une distribution conditionnelle à production non nulle. Dans ce travail, nous nous intéressons à la quantification du risque (ou de la probabilité) du puits sec. La quantification de la distribution conditionnelle à production non nulle fait l'objet d'un autre travail (Dejean et al., 1997).

La partie 1 de l'article est consacrée à la présentation de certains problèmes liés à la quantification des distributions des réponses en production, ce qui permet de situer le problème d'évaluation du risque de puits sec dans ce cadre général. Le calcul analytique de la probabilité du puits sec n'est possible que pour certains types de modèles particuliers. La partie 2 résume le calcul analytique de la probabilité du puits dans le cas des modèles booléens à grains primaires convexes, ou plus généralement dans le cas des modèles semi-markoviens (Matheron, 1967). Le but de ce travail est d'adapter la formule semi-markovienne dans les plans d'expériences pour évaluer la probabilité du puits sec d'un modèle de réservoir quelconque. On peut espérer qu'une telle démarche soit plus économique et plus précise qu'un plan d'expériences basé sur une formule de type polynomial. La méthode est testée sur trois types de modèles couramment utilisés en pratique: les modèles booléens à grains primaires non convexes, les modèles «pixel» obtenus par simulation séquentielle d'indicatrices (Deutsch et Journel, 1992) et les modèles obtenus par simulation gaussienne tronquée (Matheron et al., 1987). Les résultats de ces tests sont reportés dans la partie 3 .

\section{LA QUANTIFICATION DES INCERTITUDES ET LE RISQUE DE PUITS SEC}

\subsection{Incertitudes locales}

Un modèle stochastique de réservoir est défini par un certain nombre de paramètres statistiques (proportions de faciès, leurs longueurs de corrélation, etc.). Pour un jeu de paramètres, on peut générer de multiples réalisations ayant des comportements hydrodynamiques différents en conditions d'exploitation identiques (paramètres d'exploitation identiques). Dans ce qui suit, on notera $\vec{\theta}$ le vecteur de l'ensemble des paramètres du modèle de réservoir et des paramètres d'exploitation (longueur du puits, orientation, etc.). À $\vec{\theta}$ fixé, une réponse en production notée $Y$ est une variable aléatoire caractérisée par une fonction de répartition notée $F_{\vec{\theta}}(y) . F_{\vec{\theta}}(y)$ est appelée distribution locale car elle détermine l'incertitude de la réponse en production $Y$ pour un jeu de paramètres $\vec{\theta}$ fixé.

L'inférence statistique des paramètres d'un modèle de réservoir est souvent entachée d'incertitudes dues au nombre limité des données disponibles. En général, on se donne des intervalles de variation de ces paramètres plutôt qu'un jeu de valeurs fixes. Quant aux paramètres de production, on se donne aussi des intervalles de valeurs afin d'étudier la sensibilité des réponses en production à ces paramètres. En somme, 
nous sommes confrontés au problème de modélisation de $F_{\vec{\theta}}(y)$ pour $\vec{\theta}$ variant dans un intervalle $\left[\vec{\theta}_{\text {min }}, \vec{\theta}_{\text {max }}\right]$.

En général, $F_{\vec{\theta}}(y)$ ne peut être modélisée simplement par une loi gaussienne ou log-normale, en particulier lorsqu'un réservoir est hétérogène avec une forte proportion de faciès non-réservoir. En effet, $F_{\vec{\theta}}(y)$ présente une masse plus ou moins forte à l'origine. Si $X$ désigne l'ensemble des faciès réservoir et son complémentaire $\bar{X}$ l'ensemble des faciès nonréservoir, alors $F_{\vec{\theta}}(y)$ peut se décomposer de la façon suivante:

$$
F_{\vec{\theta}}(y)=Q_{\vec{\theta}}+\left(1-Q_{\vec{\theta}}\right) F_{\vec{\theta}}(y \mid \text { puits } \cap X \neq \varnothing)
$$

où $Q_{\vec{\theta}}=P($ puits $\subset \bar{X})$ désigne la probabilité pour que le puits soit complètement foré dans les faciès non-réservoir (probabilité du puits sec), et $F_{\vec{\theta}}(y \mid$ puits $\cap X \neq \varnothing)$ la fonction de répartition conditionnelle de la réponse en production $Y$ sachant que le puits rencontre un faciès réservoir au moins.

Dans des cas relativement simples, $F_{\vec{\theta}}(y \mid$ puits $\cap X \neq \emptyset)$ peut être modélisée par une loi monomodale (e.g. gaussienne, log-normale, etc.) paramétrée en une moyenne $M_{\vec{\theta}}$ et une variance $\Sigma_{\vec{\theta}}^{2}$. Par conséquent, la modélisation de la loi $F_{\vec{\theta}}(y)$ en fonction du vecteur des paramètres $\vec{\theta}$ revient à l'estimation de la probabilité du puits $\sec Q_{\vec{\theta}}$, de la moyenne $M_{\vec{\theta}}$ et d'une variance $\Sigma_{\vec{\theta}}^{2}$ en fonction de $\vec{\theta}$.

Il se peut que la distribution conditionnelle $F_{\vec{\theta}}(y)$ puits $\cap$ $X \neq \varnothing$ ) soit multimodale. Dans ce cas, elle ne peut être représentée par une moyenne, une variance et un type de loi simple, et nous devons encore décomposer $F_{\vec{\theta}}(y \mid$ puits $\cap$ $X \neq \varnothing)$ en des lois monomodales, en nous basant sur une analyse physique du phénomène en question (nous pensons en particulier aux problèmes liés à la connexité des corps réservoir). Jusqu'à présent, $F_{\vec{\theta}}(y \mid$ puits $\cap X \neq \emptyset)$ est modélisée par une loi gaussienne ou log-normale. La méthode des plans d'expériences est utilisée pour évaluer la moyenne $M_{\vec{\theta}}$ et la variance $\Sigma_{\vec{\theta}}^{2}$ en fonction du vecteur des paramètres $\vec{\theta}$ (Dejean et al., 1997). Dans ce qui suit, on s'intéresse uniquement à l'évaluation de la probabilité du puits $\sec Q_{\vec{\theta}}$.

\subsection{Incertitudes globales}

Dans le cadre d'un modèle stochastique de réservoir donné, les incertitudes sur les réponses en production sont engendrées par deux facteurs incertains, à savoir le vecteur des paramètres (du modèle de réservoir et d'exploitation) et le germe du modèle stochastique de réservoir. Dans ce qui précède, nous avons présenté quelques aspects de la quantification de la distribution (locale) d'une réponse en production en fonction du vecteur des paramètres $\vec{\theta}$. Il est parfois nécessaire de randomiser le vecteur des paramètres $\vec{\theta}(\vec{\theta}$ devient une réalisation d'un vecteur aléatoire noté $\vec{\Theta}$ ) afin d'aboutir à une distribution incorporant les deux facteurs incertains (appelée distribution globale et notée $F_{\vec{\Theta}}$ ). C'est en se basant sur une distribution globale que l'on obtient une évaluation des incertitudes globales.
Le choix du type de la distribution du vecteur des paramètres $\vec{\Theta}$ est plus ou moins subjectif. Si l'on considère que certains paramètres sont liés entre eux (e.g. les proportions et les longueurs de corrélation), on peut envisager de représenter la distribution de ces paramètres par une loi multigaussienne. Les autres paramètres considérés comme indépendants peuvent être représentés individuellement par des lois simples du type uniforme, triangulaire ou encore unigaussien. Une fois qu'une distribution (multivariable) de $\vec{\Theta}$ est fixée, nous pouvons obtenir une simulation de la distribution globale $F_{\vec{\Theta}}$ par la méthode de Monte-Carlo.

\section{2 ÉVALUATION DU RISQUE DE PUITS SEC: MÉTHODE}

L'évaluation du risque de puits sec s'intègre dans une procédure de quantification des incertitudes des réponses en production. Elle peut aussi aider à l'implantation d'un forage avant toute étude avancée sur les incertitudes. L'objectif de ce travail est de construire un outil permettant d'évaluer rapidement le risque de puits sec, de façon suffisamment précise, en générant un petit nombre de réalisations du modèle de réservoir. Au lieu d'employer la méthode des plans d'expériences basée sur une approximation polynomiale, il semble a priori plus avantageux d'utiliser une formule issue d'un modèle de réservoir sur lequel le calcul analytique de la probabilité du puits sec est possible, ce qui nous amène à considérer le modèle booléen.

\subsection{Modèle booléen à grains primaires convexes}

Soit $A$ un objet élémentaire aléatoire (appelé aussi grain primaire). La construction d'un modèle booléen consiste à générer des objets élémentaires indépendants $A_{i}(i=1,2, \ldots)$ ayant les mêmes caractéristiques statistiques que l'objet $A$, et ensuite à les implanter dans l'espace au hasard, indépendamment les uns des autres. L'union de ces objets, notée $X$, constitue un ensemble aléatoire appelé modèle booléen:

$$
X=\cup_{i} A_{i}
$$

La probabilité pour qu'un objet $B$ soit contenu dans le complémentaire de $X$ est donnée par:

$$
P(B \subset \bar{X})=q^{\frac{E|A \oplus \check{B}|}{E|A|}}
$$

où $q$ est la proportion du volume occupé $\operatorname{par} \bar{X}$. $|A|$ désigne le volume de $A$ et $|A \oplus \stackrel{\vee}{ }|$ le volume de $A$ dilaté par $B$ (Matheron, 1967).

Considérons maintenant que $X$ représente l'ensemble des faciès réservoir et son complémentaire $\bar{X}$ l'ensemble des faciès non-réservoir. Prenons un segment $l_{\alpha}$ de longueur $l$ et d'orientation $\alpha$ et considérons que $l_{\alpha}$ représente un puits. 
Si l'objet élémentaire $A$ est convexe, alors:

$$
\left|A \oplus \check{l}_{\alpha}\right|=|A|+l\left|A_{\alpha}\right|
$$

où $\left|A_{\alpha}\right|$ désigne la surface apparente (largeur apparente en 2D) de $A$ dans la direction $\alpha$ (fig. 1).

On obtient ensuite aisément la probabilité pour que $l_{\alpha}$ soit contenu dans l'ensemble des faciès non-réservoir (probabilité du puits sec):

$$
P\left(l_{\alpha} \subset \bar{X}\right)=q e^{\frac{l}{q} C_{\alpha}^{\prime}(0)}
$$

où $C_{\alpha}^{\prime}(0)$ est la dérivée (pente) à l'origine de la covariance du faciès réservoir (ou non-réservoir) dans la direction $\alpha$.

Pour une covariance exponentielle de portée pratique $a_{\alpha}$ dans la direction $\alpha$, on a:

$$
\mathrm{C}_{\alpha}^{\prime}(0)=-3 q(1-q) / a_{\alpha}
$$

Pour une covariance sphérique de portée $a_{\alpha}$ dans la direction $\alpha$, on a :

$$
\mathrm{C}_{\alpha}^{\prime}(0)=-1,5 q(1-q) / a_{\alpha}
$$

\subsection{Extension vers $d$ 'autres modèles}

La formule pour le calcul de la probabilité du puits sec obtenue dans le cadre des modèles booléens à grains primaires convexes reste valable pour une classe plus générale de modèles vérifiant la propriété semi-markovienne. Nous rappelons ici la définition de la propriété semi-markovienne, bien que ceci ne soit pas essentiel pour la lecture de la suite de cet article.

Étant donné trois ensembles $E_{1}, E_{2}$ et $C$, nous dirons que $C$ sépare $E_{1}$ et $E_{2}$ si tout segment $[x, y]$ joignant un point $x$ de $E_{1}$ et un point $y$ de $E_{2}$ rencontre $C$ en un point au moins (situé entre $x$ et $y$ ) (fig. 2). Nous dirons qu'un ensemble aléatoire $X$ est semi-markovien si, quels que soient les ensembles $E_{1}, E_{2}$ et $C$ séparant $E_{1}$ et $E_{2}$, il y a indépendance conditionnelle entre tout événement intéressant $E_{1}$ et tout événement intéressant $E_{2}$, lorsque l'on sait que $C$ est contenu dans $\bar{X}$ (complémentaire de $X$ ).

On peut montrer que les modèles booléens à grains primaires convexes vérifient la propriété semi-markovienne. En revanche, les modèles booléens à grains primaires non convexes ne sont pas semi-markoviens. Dans les applications pétrolières, les modèles géologiques sont souvent construits avec des objets élémentaires de forme non convexe (e.g. chenaux, dunes sous forme de croissant, etc.). La formule (1) n'est donc pas directement applicable à ces cas. En effet, l'idée de départ était de remplacer une formule polynomiale, souvent utilisée dans les plans d'expériences, par une formule plus appropriée pour évaluer la probabilité du puits sec. Comme pour les formules polynomiales des plans d'expériences, on peut introduire des coefficients dans la formule (1) (que nous appellerons formule semi-markovienne); ces coefficients sont à déterminer à partir des données issues d'un plan d'expériences. Une telle démarche est normalement plus économique et donne des prédictions plus précises qu'un plan d'expériences basé sur une approximation polynomiale.

La première possibilité de modifier la formule (1) consiste à introduire un coefficient dans son exposant. Nous obtenons ainsi la formule suivante pour évaluer la probabilité du puits sec:

$$
P\left(l_{\alpha} \subset \bar{X}\right)=q e^{-\lambda p \frac{l}{L \alpha}}
$$

où $p=1-q$ désigne la proportion du faciès réservoir et $L_{\alpha}$ sa longueur de corrélation dans la direction $\alpha$ (équivalente à la porté du variogramme à un facteur multiplicatif près). On vérifie immédiatement que si la longueur du puits est très

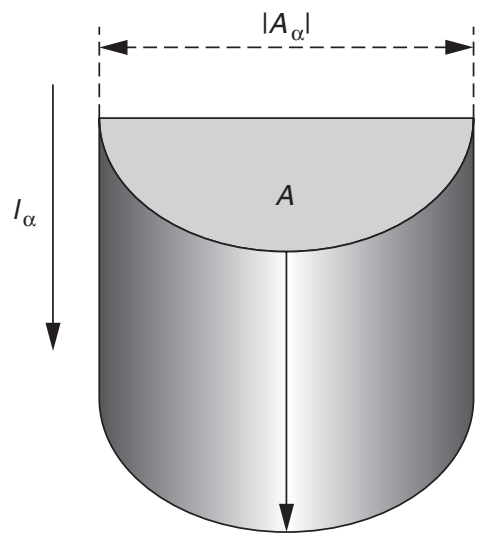

Figure 1

Dilatation d'un objet convexe par un segment. Dilatation of a convex object by a segment.

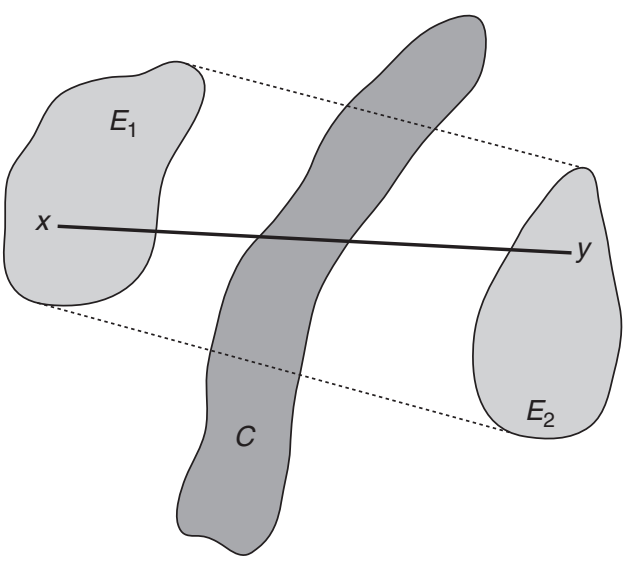

Figure 2

Les ensembles $E_{1}$ et $E_{2}$ séparés par l'ensemble $C$. Sets $E_{1}$ and $E_{2}$ separated by set $C$. 
faible (tend vers zéro), on retrouve la proportion du faciès non-réservoir. Le coefficient est à ajuster sur chaque modèle de réservoir utilisé. En principe, il suffit d'avoir une seule donnée de probabilité du puits sec (obtenue à partir d'une seule réalisation du modèle de réservoir) pour déterminer le coefficient $\lambda$. En pratique, il est préférable de générer plusieurs réalisations (avec des paramètres différents) selon un plan d'expériences pour obtenir une valeur de $\lambda$ globalement satisfaisante. D'ailleurs, l'utilisation de plusieurs réalisations permet de vérifier la validité de la formule (2) dans l'ensemble du domaine d'expérimentation.

Le chapitre suivant est consacré à l'application de la formule (2) dans trois cas différents.

\section{3 ÉVALUATION DU RISQUE DE PUITS SEC: VALIDATION}

Le premier cas considéré est celui d'un modèle booléen à grains primaires non convexes. De nombreuses variantes de ce modèle (dit modèle objet) sont utilisées en pratique pour la modélisation des corps réservoir. Les méthodes dites pixel sont aussi souvent utilisées pour la modélisation de réservoirs. Nous considérons ensuite les modèles générés par deux méthodes pixel couramment utilisées en pratique: la méthode de simulation séquentielle d'indicatrices et la méthode de simulation gaussienne tronquée.

\subsection{Modèle booléen à grains primaires non convexes}

Soit un objet $A$ ayant une forme non convexe présentée dans la figure 3. Lorsqu'un segment (puits) $l_{\alpha}$ ne peut rencontrer qu'une fois $A$, le volume de $A$ dilaté par $l_{\alpha}$ s'écrit:

$$
\left|A \oplus \check{l}_{\alpha}\right|=|A|+l\left(\left|A_{\alpha}^{1}\right|+\left|A_{\alpha}^{2}\right|\right)
$$

où $\left|A_{\alpha}^{1}\right|$ et $\left|A_{\alpha}^{2}\right|$ désignent respectivement les surfaces apparentes (largeurs apparentes en 2D) extérieure et intérieure de $A$ dans la direction $\alpha$. À partir de cette expression, il est aisé de vérifier que la formule (1) reste valable sans aucune modification. Dans les applications pétrolières, lorsqu'un puits est vertical ou subvertical, il ne peut traverser plus d'une fois un chenal ou une dune. La formule (1) peut être directement appliquée si les chenaux ou les dunes sont implantés au hasard dans l'espace. En revanche, si le mode de construction n'est pas tout à fait celui d'un modèle booléen, on sera amené à utiliser plutôt la formule modifiée (2) en ajustant le paramètre $\lambda$.

Revenons au cas de modèles booléens à grains primaires non convexes. Si maintenant le segment $l_{\alpha}$ peut rencontrer plus d'une fois l'objet $A$, alors il n'y a plus de formule générale pour calculer la probabilité du puits sec en fonction de la proportion du faciès non-réservoir, de la longueur de corrélation du faciès non-réservoir, de la longueur et de l'orientation du puits. En effet, la probabilité du puits sec dépend de la forme particulière de l'objet élémentaire.
L'exemple qui suit illustre la différence entre la formule semi-markovienne (1) et la formule exacte de la probabilité du puits sec dans le cas d'un objet élémentaire non convexe de forme très simple (fig. 4). Des objets identiques de cette forme sont implantés dans l'espace pour construire un modèle de réservoir booléen à $2 \mathrm{D}$. Pour un puits de longueur $l$ orienté dans la direction $\alpha$ parallèle à l'allongement des

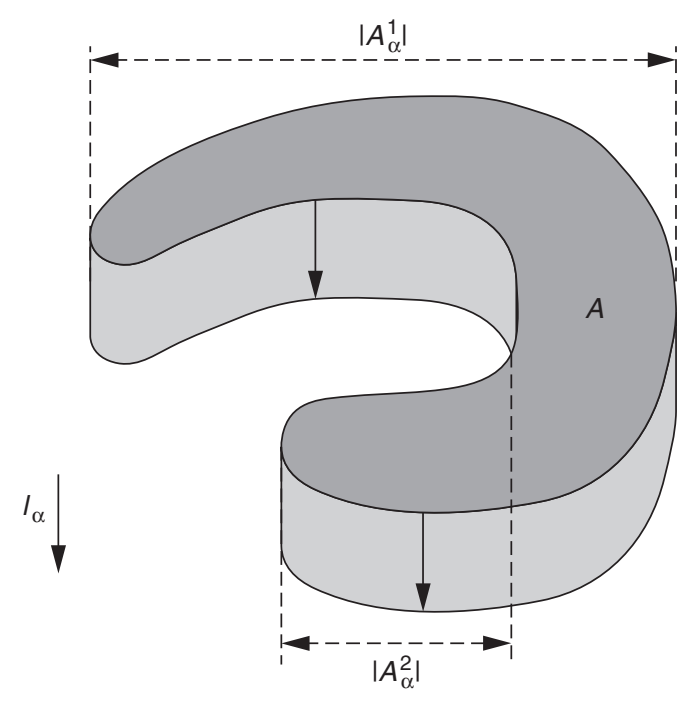

Figure 3

Dilatation d'un objet non convexe par un segment. Dilatation of a nonconvex object by a segment.

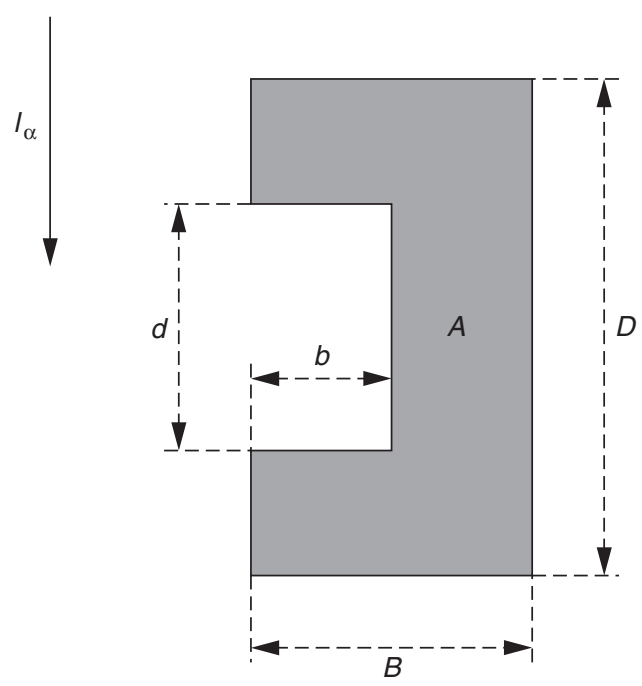

Figure 4

Objet élémentaire non convexe pour la construction d'un modèle booléen.

Elementary nonconvex object for the construction of a Boolean model. 
dimensions $D$ et $d$, on obtient les expressions exactes de la probabilité du puits sec:

$$
\begin{array}{ll}
Q=P\left(l_{\alpha} \subset \bar{X}\right)=q q^{\frac{B+b}{B D+b d} l} & (l<d) \\
Q=P\left(l_{\alpha} \subset \bar{X}\right)=q q^{\frac{B l+b d}{B D-b d} l} \quad(l>d)
\end{array}
$$

Il est aisé de vérifier que l'expression ci-dessus est identique à la formule (1) dans le cas $l<d$. En effet, on a:

$$
C_{\alpha}^{\prime}(0)=\frac{B+b}{B D-b d} q \ln q
$$

En revanche, dans le cas $l>d$, cette expression est différente de la formule (1). La figure 5 illustre cette différence pour un jeu fixé des paramètres $q, B, b, D$ et $d$ et un puits de longueur variable. On note par exemple que pour une probabilité du puits sec égale à 30\%, la formule semi-markovienne prévoit seulement $20 \%$.

Au lieu de la formule semi-markovienne sous sa forme initiale (1), la formule (2) est ensuite utilisée. L'ajustement du coefficient $\lambda$ à partir d'une valeur de la probabilité du puits sec expérimentale $(Q=0,304, l=6)$ donne une valeur de $\lambda$ égale à 0,722 . La figure 6 montre qu'un tel ajustement de la formule (2) donne des prédictions globalement satisfaisantes.

\subsection{Modèle généré par simulation séquentielle d'indicatrices}

La méthode de simulation séquentielle d'indicatrices a été utilisée pour construire des modèles en faciès d'un gisement synthétique nommé Calamity Jane. Le mode de construction de ces modèles et le choix de leurs paramètres ont été inspirés d'un gisement réel d'Elf. Les modèles sont composés de trois faciès, dont un faciès non-réservoir (faciès mur) et deux faciès réservoir (faciès drain et faciès matrice). Le gisement Calamity Jane a permis de tester la sensibilité de l'indice de productivité (IP) d'un puits horizontal aux incertitudes sur les paramètres du modèle de réservoir et ceux d'exploitation. Le simulateur d'essai de puits SIMTEST est utilisé pour déterminer les valeurs de l'IP (Blanc, 1997). Un fort taux de valeurs de l'IP quasiment nulles a été observé, car la proportion du faciès non-réservoir (faciès mur) est dominante dans les modèles, et cela conduit à une probabilité considérable pour que le puits soit complètement foré dans le faciès nonréservoir. Il a été décidé de traiter séparément le problème du puits sec et de calculer l'IP en conditionnant le puits par un faciès réservoir (le puits rencontre au moins un point du faciès réservoir).

Le programme SISIMPDF (algorithme de simulation séquentielle d'indicatrices de la GSLIB (Deutsch et Journel, 1992)) est utilisé pour générer les réalisations. La covariance

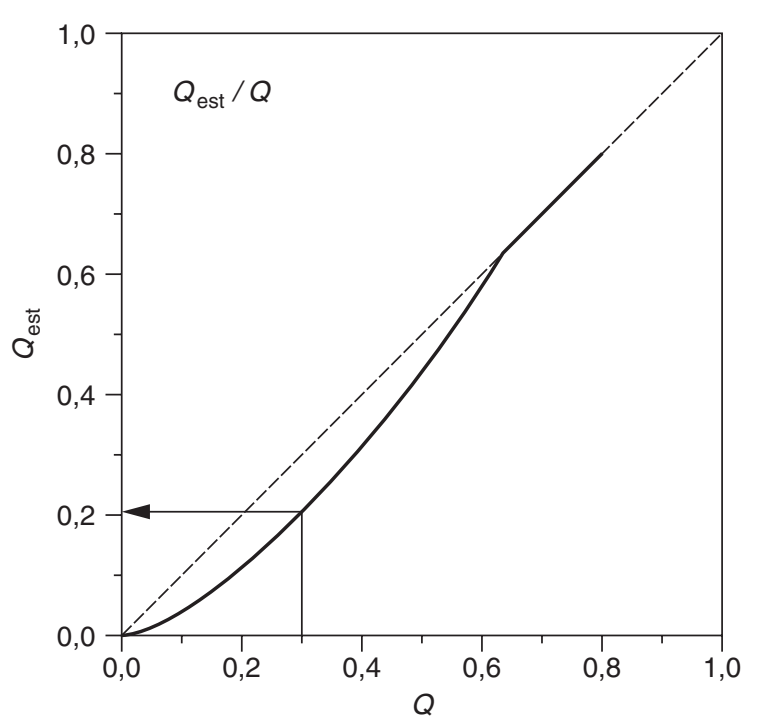

Figure 5

Comparaison entre les probabilités du puits sec expérimentales $(Q)$ et celles estimées $\left(Q_{\text {est }}\right)$ par la formule (1) avec $q=0,8, B=1, b=0,5, D=2$ et $d=1$.

Comparison between the real dry well probability $(Q)$ and the estimated dry well probability $\left(Q_{\text {est }}\right)$ using Formula (1) with $q=0.8, B=1, b=0.5, D=2$ and $d=1$.

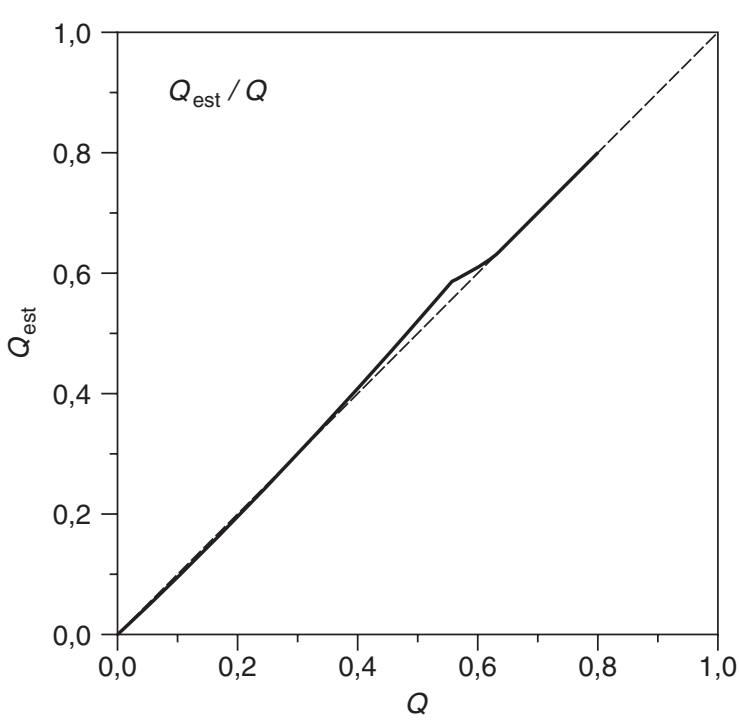

Figure 6

Comparaison entre les probabilités du puits sec expérimentales $(Q)$ et celles estimées $\left(Q_{\text {est }}\right)$ par la formule (2) avec $\lambda=0,722$ et avec $q=0,8, B=1, b=0,5, D=2$ et $d=1$.

Comparison between the real dry well probability $(Q)$ and the estimated dry well probability $\left(Q_{\mathrm{est}}\right)$ using Formula (2) with $\lambda=0.722$ and with $q=0.8, B=1, b=0.5, D=2$ and $d=1$. 
exponentielle est utilisée (l'expérience montre que l'utilisation d'une covariance exponentielle par SISIMPDF permet une bonne reproduction des paramètres d'entrée, contrairement à d'autres types de covariance). Les paramètres influant sur la probabilité du puits sec sont la proportion du faciès mur (faciès non-réservoir), la longueur de corrélation du faciès mur dans la direction d'allongement du puits et la longueur du puits. Le tableau 1 montre les valeurs minimales, moyennes et maximales de ces paramètres.

TABLEAU 1

Cas Calamity Jane: valeurs minimales, moyennes et maximales des paramètres du modèle géostatistique

Calamity Jane case: minimal, mean and maximal values of the parameters of the geostatistical model

\begin{tabular}{l|c|c|c}
\hline & $\begin{array}{c}\text { Valeur } \\
\text { minimale }\end{array}$ & $\begin{array}{c}\text { Valeur } \\
\text { moyenne }\end{array}$ & $\begin{array}{c}\text { Valeur } \\
\text { maximale }\end{array}$ \\
\hline $\begin{array}{l}\text { Proportion du faciès } \\
\text { non-réservoir (\%) }\end{array}$ & 72 & 78 & 84 \\
$\begin{array}{l}\text { Longueur de corrélation } \\
\text { dans l'orientation du puits } \\
(1 / 3 \text { de la portée pratique) (m) }\end{array}$ & 75 & 100 & 125 \\
Longueur du puits (m) & 270 & 510 & 750
\end{tabular}

Le tableau 2 montre les valeurs expérimentales des probabilités du puits sec pour les 27 combinaisons des valeurs des trois paramètres. Ces valeurs sont obtenues par balayage des différentes réalisations avec des segments de longueurs variées.

La formule semi-markovienne (1) est employée pour calculer les probabilités du puits sec dans les 27 cas. Lorsqu'on compare les valeurs ainsi obtenues avec celles expérimentales, on constate une surestimation très importante dans l'ensemble des 27 cas (fig. 7). Cela montre que les modèles

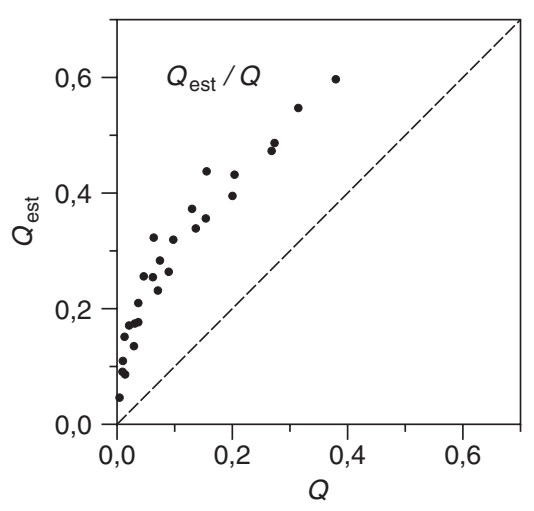

Figure 7

Simulations séquentielles d'indicatrices: comparaison entre les probabilités du puits sec expérimentales $(Q)$ et celles estimées $\left(Q_{\text {est }}\right)$ par la formule (1).

Sequential indicator simulations: comparison between the real dry well probability $(Q)$ and the estimated dry well probability $\left(Q_{\mathrm{est}}\right)$ using Formula $(1)$.
TABLEAU 2

Simulations séquentielles d'indicatrices: valeurs des probabilités du puits sec

Sequential indicator simulations: values of the dry well probability

\begin{tabular}{|c|c|c|c|c|}
\hline $\begin{array}{c}\mathrm{N}^{\circ} \\
\text { du cas }\end{array}$ & $\begin{array}{c}\text { Proportion } \\
\text { (\%) }\end{array}$ & $\begin{array}{l}\text { Longueur de } \\
\text { corrélation }(\mathrm{m})\end{array}$ & $\begin{array}{l}\text { Longueur } \\
\text { du puits (m) }\end{array}$ & $\begin{array}{l}\text { Probabilité } \\
\text { du puits sec }\end{array}$ \\
\hline 1 & 72 & 75 & 270 & 0,09033 \\
\hline 2 & 72 & 75 & 510 & 0,00998 \\
\hline 3 & 72 & 75 & 750 & 0,00153 \\
\hline 4 & 72 & 100 & 270 & 0,13622 \\
\hline 5 & 72 & 100 & 510 & 0,02992 \\
\hline 6 & 72 & 100 & 750 & 0,01031 \\
\hline 7 & 72 & 125 & 270 & 0,20195 \\
\hline 8 & 72 & 125 & 510 & 0,07109 \\
\hline 9 & 72 & 125 & 750 & 0,03095 \\
\hline 10 & 78 & 75 & 270 & 0,15530 \\
\hline 11 & 78 & 75 & 510 & 0,03722 \\
\hline 12 & 78 & 75 & 750 & 0,01252 \\
\hline 13 & 78 & 100 & 270 & 0,20388 \\
\hline 14 & 78 & 100 & 510 & 0,04733 \\
\hline 15 & 78 & 100 & 750 & 0,01316 \\
\hline 16 & 78 & 125 & 270 & 0,27397 \\
\hline 17 & 78 & 125 & 510 & 0,09823 \\
\hline 18 & 78 & 125 & 750 & 0,03813 \\
\hline 19 & 84 & 75 & 270 & 0,26894 \\
\hline 20 & 84 & 75 & 510 & 0,07436 \\
\hline 21 & 84 & 75 & 750 & 0,02116 \\
\hline 22 & 84 & 100 & 270 & 0,31537 \\
\hline 23 & 84 & 100 & 510 & 0,13060 \\
\hline 24 & 84 & 100 & 750 & 0,06228 \\
\hline 25 & 84 & 125 & 270 & 0,38019 \\
\hline 26 & 84 & 125 & 510 & 0,15630 \\
\hline 27 & 84 & 125 & 750 & 0,06425 \\
\hline
\end{tabular}


du cas Calamity Jane sont incompatibles avec la propriété semi-markovienne ou, plus particulièrement, avec un modèle booléen à grains primaires convexes, ce qui n'est d'ailleurs pas étonnant étant donné la méthode de construction employée dans ce cas.

La validité de la formule (2) est testée dans ce cas. L'ajustement du paramètre $\lambda$ donne une valeur de 2,2 et l'estimation de la probabilité du puits sec est satisfaisante pour l'ensemble des 27 cas (fig. 8).

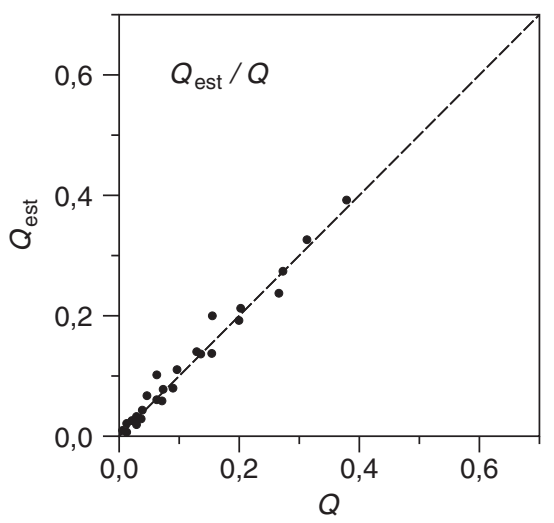

Figure 8

Simulations séquentielles d'indicatrices: comparaison entre les probabilités du puits sec expérimentales $(Q)$ et celles estimées $\left(Q_{\text {est }}\right)$ par la formule (2) avec $1=2,2$.

Sequential indicator simulations: comparison between the real dry well probability $(Q)$ and the estimated dry well probability $\left(Q_{\mathrm{est}}\right)$ using Formula (2) with $l=2.2$

\subsection{Modèle généré par simulation gaussienne tronquée}

La méthode de simulation gaussienne tronquée consiste à tronquer une réalisation d'une fonction aléatoire multigaussienne avec différents seuils pour obtenir une représentation lithologique en 3D des réservoirs. Pour tester la validité de l'approximation semi-markovienne de la probabilité du puits sec pour des modèles gaussiens tronqués, considérons le cas simple d'un modèle gaussien tronqué stationnaire composé de deux faciès (réservoir et non-réservoir). La covariance de la fonction aléatoire gaussienne est choisie exponentielle. Le seuil de troncature est déterminé par la proportion du faciès non-réservoir. La probabilité du puits sec est une fonction de la proportion du faciès non-réservoir, de la longueur de corrélation de la fonction aléatoire gaussienne dans la direction d'allongement du puits, et de la longueur du puits. Notons que c'est la longueur de corrélation de la fonction aléatoire gaussienne qui est utilisée plutôt que celle des faciès, car c'est la première qui est directement nécessaire lors de la génération d'un modèle gaussien tronqué. Lorsque la proportion est fixée, la longueur de corrélation de la gaussienne est entièrement déterminée par celle du faciès.

Le tableau 3 montre des valeurs expérimentales des probabilités du puits sec pour différents jeux de paramètres. Ces valeurs sont obtenues sur un grand nombre de réalisations indépendantes de vecteurs multigaussiens le long du puits.

\section{TABLEAU 3}

Simulations gaussiennes tronquées: valeurs des probabilités du puits sec

Truncated Gaussian simulations: values of the dry well probability

\begin{tabular}{|c|c|c|c|c|}
\hline $\begin{array}{c}\mathrm{N}^{\circ} \\
\text { du cas }\end{array}$ & $\begin{array}{c}\text { Proportion } \\
(\%)\end{array}$ & $\begin{array}{l}\text { Longueur de } \\
\text { corrélation (m) }\end{array}$ & $\begin{array}{c}\text { Longueur } \\
\text { du puits (m) }\end{array}$ & $\begin{array}{l}\text { Probabilité } \\
\text { du puits sec }\end{array}$ \\
\hline 1 & 72 & 75 & 270 & 0,1625 \\
\hline 2 & 72 & 125 & 270 & 0,2535 \\
\hline 3 & 72 & 75 & 750 & 0,0117 \\
\hline 4 & 72 & 125 & 750 & 0,0455 \\
\hline 5 & 72 & 100 & 510 & 0,0748 \\
\hline 6 & 78 & 75 & 510 & 0,0810 \\
\hline 7 & 78 & 125 & 510 & 0,1665 \\
\hline 8 & 78 & 100 & 270 & 0,2915 \\
\hline 9 & 78 & 100 & 750 & 0,0542 \\
\hline 10 & 78 & 100 & 510 & 0,1258 \\
\hline 11 & 84 & 75 & 270 & 0,3385 \\
\hline 12 & 84 & 125 & 270 & 0,4405 \\
\hline 13 & 84 & 75 & 750 & 0,0682 \\
\hline 14 & 84 & 125 & 750 & 0,1505 \\
\hline 15 & 84 & 100 & 510 & 0,2087 \\
\hline 16 & 50 & 100 & 200 & 0,1058 \\
\hline 17 & 98 & 100 & 900 & 0,6323 \\
\hline 18 & 95 & 100 & 30 & 0,9285 \\
\hline 19 & 100 & 100 & 510 & 1,0000 \\
\hline 20 & 0 & 100 & 510 & 0,0000 \\
\hline
\end{tabular}

Comme dans le cas des simulations séquentielle d'indicatrices, la formule semi-markovienne (1) est d'abord employée pour calculer les probabilités du puits sec avec ces 20 jeux de paramètres du cas gaussien tronqué. On obtient sans surprise un biais systématique de ces estimations par rapport aux valeurs expérimentales (fig. 9), car les modèles gaussiens tronqués ne sont pas semi-markoviens. Enfin, en utilisant la formule (2) avec un ajustement du paramètre $\lambda(=1,8)$, les estimations sont tout à fait satisfaisantes (fig. 10). 


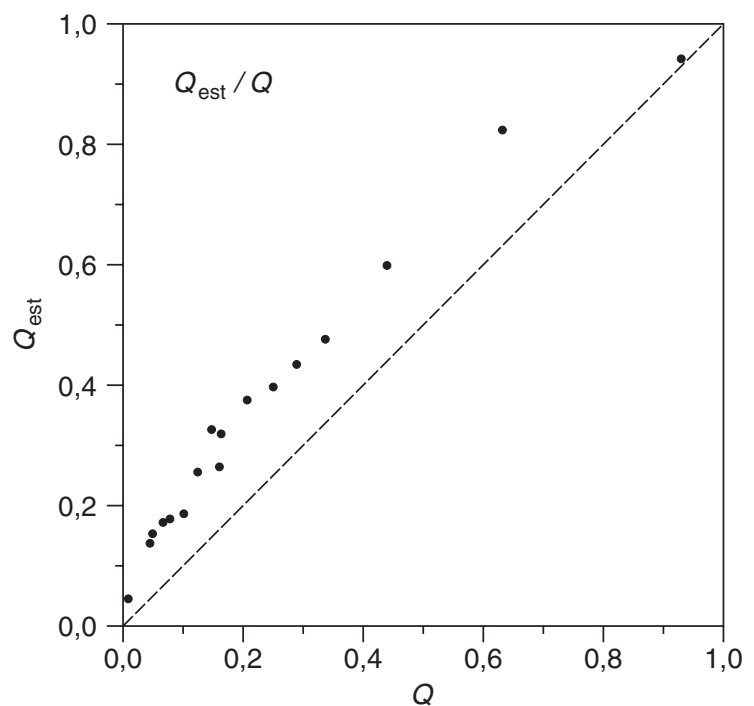

Figure 9

Simulations gaussiennes tronquées: comparaison entre les probabilités du puits sec expérimentales $(Q)$ et celles estimées $\left(Q_{\text {est }}\right)$ par la formule (1).

Truncated Gaussian simulations: comparison between the real dry well probability $(Q)$ and the estimated dry well probability $\left(Q_{\text {est }}\right)$ using Formula (1).

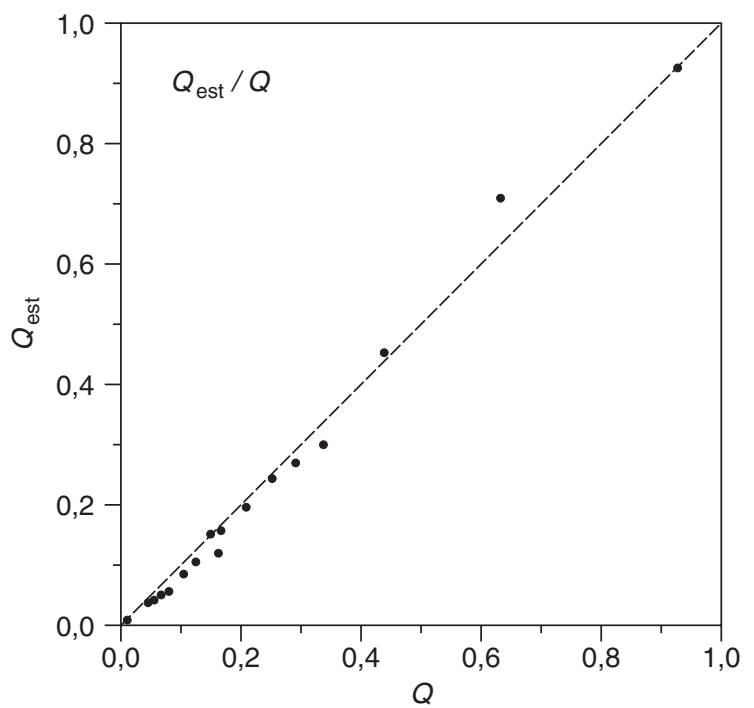

Figure 10

Simulations gaussiennes tronquées: comparaison entre les probabilités du puits sec expérimentales $(Q)$ et celles estimées $\left(Q_{\text {est }}\right)$ par la formule (2) avec $\lambda=1,8$.

Truncated Gaussian simulations: comparison between the real dry well probability $(Q)$ and the estimated dry well probability $\left(Q_{\text {est }}\right)$ using Formula (2) with $\lambda=1.8$.

\section{DISCUSSION ET CONCLUSIONS}

Dans le cas d'un réservoir hétérogène avec une forte proportion de faciès non-réservoir, le risque de puits sec peut être considérable. L'évaluation de ce risque (estimation de la probabilité du puits sec) est, d'une part, nécessaire avant la prise de décision d'implantation d'un puits. D'autre part, elle fait partie d'une procédure de quantification des incertitudes sur les réponses en production d'un puits. Nous avons présenté comment l'évaluation de probabilité du puits sec s'intègre dans cette procédure.

Il convient de mentionner que cette procédure n'inclut pas tous les aspects incertains du processus de caractérisation et de simulation de réservoirs. En effet, on se limite à la quantification des incertitudes dans le cadre d'un modèle stochastique de réservoir préalablement adopté (selon des critères géologiques par exemple). Le choix d'un modèle est souvent entaché de subjectivité et donc d'incertitude. Mais cet aspect d'incertitude est difficilement quantifiable. Toutefois, on peut penser que l'incertitude sur le choix d'un modèle est partiellement prise en compte par le fait que l'on fait varier les paramètres du modèle choisi. Une fois qu'un modèle de réservoir est adopté, la quantification des incertitudes consiste à établir la distribution de chaque réponse en production, d'abord localement en fonction des paramètres du modèle de réservoir et d'exploitation, et ensuite globalement en fixant a priori une distribution (multivariable) des paramètres.

Afin d'évaluer la probabilité du puits sec dans le cadre d'un modèle stochastique de réservoir, nous proposons, dans cet article, une méthode de régression basée sur les modèles booléens à grains primaires convexes (ou plus généralement la classe de modèles ayant la propriété semi-markovienne). Bien que cette méthode soit spécifique à une classe de modèles particulière, nous pensons qu'elle est mieux adaptée à l'évaluation de la probabilité du puits sec que des régressions du type polynomial. Cette méthode est validée par trois exemples de modèles (non semi-markoviens) construits par des méthodes couramment utilisées en pratique (méthode booléenne à grains primaires non convexes, simulation séquentielle d'indicatrices et simulation gaussienne tronquée). Dans ces exemples, un seul coefficient est introduit dans la formule de régression. En principe, une seule expérience suffit pour déterminer ce coefficient. En pratique, il est préférable ou même nécessaire de faire plus d'une expérience (et ceci, avec des jeux de paramètres selon un plan d'expériences) pour contrôler la validité de la méthode proposée. Si un seul coefficient s'avérait insuffisant pour l'ensemble du domaine d'expérimentation (l'espace des paramètres), il serait alors nécessaire d'introduire d'autres coefficients. 


\section{REMERCIEMENTS}

Ce travail est réalisé dans le cadre du projet commun Elf Exploration \& Production et Institut français du pétrole.

\section{RÉFÉRENCES}

Blanc, G. (1997) Évaluation des performances des puits. Indices de productivité. Application au gisement CJ. Rapport du projet commun IFP/Elf en gisement, réf. IFP: 43744.

Dejean, J.P., Morelon, I. et Piacentino, L. (1997) Étude de sensibilité de l'indice de productivité d'un puits horizontal aux incertitudes sur les paramètres géostatistiques et les paramètres d'exploitation. Gisement Calamity Jane. Rapport du projet commun IFP/Elf en gisement, réf. IFP: 43746.

Deutsch, C. et Journel, A. (1992) GSLIB, Geostatistical Software Library and User's Guide, Oxford University Press, New York, Oxford.

Matheron, G. (1967) Éléments pour une théorie des milieux poreux, Masson, Paris.

Matheron, G., Beucher, H., de Fouquet, C., Galli, A. et Ravenne, C. (1987) Conditional Simulation of the Geometry of FluvioDeltaic Reservoirs. SPE 16753.

Manuscrit définitif reçu en décembre 1999 